\title{
Levothyroxine Administration during Ramadan: A Prospective Randomized Controlled Trial
}

\author{
Samer El-Kaissi ${ }^{a} \quad$ Laila AbdelWareth $^{b}$ Ruba Dajanic ${ }^{c}$ Terrence J. Lee-St. John ${ }^{d}$ \\ Sherry Ann Santarinad ${ }^{d}$ Fiona Makiad $^{d}$ Malak AlTakruric AbedEIRahman Kaskas ${ }^{\mathrm{e}}$ \\ Yahya Ahmed ${ }^{\mathrm{c}}$ \\ aDepartment of Endocrinology, Medical Subspecialties Institute, Cleveland Clinic Abu Dhabi, Abu Dhabi, UAE; \\ ${ }^{b}$ Department of Laboratory Medicine, National Reference Laboratory and Cleveland Clinic Abu Dhabi, Abu Dhabi, UAE;

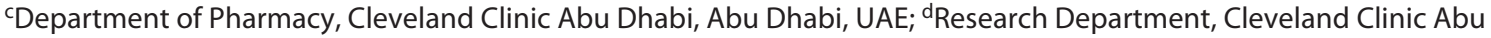 \\ Dhabi, Abu Dhabi, UAE; 'Department of Patient Education, Cleveland Clinic Abu Dhabi, Abu Dhabi, UAE
}

\section{Keywords}

Thyroid $\cdot$ Hypothyroidism $\cdot$ Levothyroxine $\cdot$ Ramadan $\cdot$

Fasting

\section{Abstract}

Background and Aim: We have previously shown in a retrospective analysis that the plasma thyroid-stimulating hormone (TSH) rises significantly post-Ramadan in levothyroxine-treated hypothyroid patients, possibly as a result of lifestyle alterations and time restrictions during the nonfasting period from dusk until dawn. The aim of this study is to determine the best time to instruct patients to take levothyroxine during Ramadan so as to minimize changes in thyroid function tests during this period. Methods: In a randomized prospective design, hypothyroid patients taking levothyroxine were randomized to receive instructions to take levothyroxine at one of the following 3 times during Ramadan: (group 1) at dusk 30-min before Iftar meal, (group 2) 3 or more hours after Iftar meal, or (group 3) at dawn 30-min before Suhur meal. Thyroid function tests were performed within 3 months before Ramadan and within 6 weeks postRamadan. Data from patients with at least 1 blood test be- fore or after Ramadan were analyzed using mixed-effects regression models. Results: Plasma TSH levels were available at one or more time points for 148 patients, group $1(n=50)$, group $2(n=46)$, and group $3(n=52)$. A statistically significant within-patient increase in plasma TSH was seen in patients at the 25th percentile pre-Ramadan in groups 2 and 3 ( $p$ values $<0.001$ ), but not in group 1 . A statistically significant within-patient decrease in plasma TSH was found in patients at the 75th percentile in group 1 only. For patients at the 50th percentile pre-Ramadan, no statically significant within-patient changes were found, though descriptively, increases in plasma TSH were observed for groups 2 and 3, while a decrease was observed in group 1. Conclusions: Our data suggest that instructing patients to take levothyroxine at the time of breaking the fast $30 \mathrm{~min}$ before the Iftar meal minimizes unfavorable changes in plasma TSH post-Ramadan. In contrast, instructing patients to take levothyroxine $3 \mathrm{~h}$ post-Iftar or $30 \mathrm{~min}$ before Suhur led to a greater rise in post-Ramadan TSH.

(c) 2021 European Thyroid Association Published by S. Karger AG, Basel

This study was conducted at Cleveland Clinic Abu Dhabi, Al Maryah Island, Abu Dhabi, UAE.
Correspondence to:

Samer El-Kaissi, elkaiss@ clevelandclinicabudhabi.ae 


\section{Introduction}

The holy month of Ramadan is the 9th lunar month of the Islamic Hijri calendar, and fasting from dawn until dusk is observed by Muslims during this month. While there are wide variations in the eating habits during Ramadan, most people consume 2 meals, the main meal or "Iftar" at dusk and a smaller meal at dawn "Suhur."

The altered eating habits during Ramadan, in addition to time constraints during the nonfasting period, present a challenge for levothyroxine-treated patients and may lead to reduced compliance with the instructions of taking levothyroxine on an empty stomach and at least 30 min before eating and drinking, which in turn may lead to an increase in plasma thyroid-stimulating hormone (TSH) post-Ramadan, as demonstrated in a number of prospective studies [1-4] and by our group in a retrospective analysis [5]. However, it is unknown if the change in thyroid function in Ramadan is associated with worsening symptoms of hypothyroidism.

Levothyroxine is absorbed in the jejunum and ileum, and its absorption is enhanced by an acidic environment in the stomach as occurs in the fasting state. Approximately $80 \%$ of the administered dose is absorbed under ideal fasting conditions [6], compared to about $64 \%$ absorption when the medication is taken with food [7].

There are currently no specific recommendations for the administration of levothyroxine during Ramadan. The American Thyroid Association (ATA) guidelines for the treatment of hypothyroidism recommend that levothyroxine generally be taken either $60 \mathrm{~min}$ before breakfast or at bedtime at least $3 \mathrm{~h}$ after the evening meal [8]. This study aims to determine the best time to instruct patients to take levothyroxine during Ramadan so as to minimize changes in thyroid function tests.

\section{Methods}

In a prospective randomized trial, adult patients with hypothyroidism treated with levothyroxine for $>6$ months and planning to fast during Ramadan were randomized to receive instructions to take levothyroxine at one of 3 times: (group 1) at the time of breaking the fast at dusk 30 min before the main Iftar meal, (group 2) 3 or more hours after Iftar meal, or (group 3) at dawn 30 min before Suhur meal (see online suppl. Fig. 1; see online Supplementary Materials). Patients in groups (2) and (3) were instructed to refrain from eating and drinking for at least $3 \mathrm{~h}$ before and $30 \mathrm{~min}$ after taking levothyroxine. Randomization into the 3 groups was performed using equal-probability assignment: a randomly drawn number form a uniform distribution determined each participant's group assignment with the equal probabilities of assignment being 0.3333 .
After group assignment, levothyroxine administration instructions were provided verbally by telephone, and a text message was sent to patients midway through Ramadan as a reminder to adhere to the instructions. At the end of Ramadan, patients were contacted by telephone to assess the number of days fasted, compliance with levothyroxine as per randomization, and satisfaction with the assigned time for taking levothyroxine.

Thyroid function tests were performed within 3 months before Ramadan and repeated within 6 weeks post-Ramadan [8]. Patients younger than 18 years and those unable to provide an informed consent were excluded. In an attempt to use a sample representative of the population of patients on levothyroxine, we did not exclude pregnant women, patients who had a change of levothyroxine dose, or those taking medications that may interfere with levothyroxine absorption. Although including patients with potential confounding factors may affect study outcomes if these variables are unevenly distributed across groups, the randomized design of the study protects against such threats to internal validity.

Plasma TSH and free thyroxine (free-T4) were measured with the Roche e601 immunoassay analyzer using the electrochemiluminescence immunoassay sandwich principle for TSH and the electrochemiluminescence immunoassay competition principle for free-T4.

The study was conducted during Ramadan 2019 at a tertiary health care facility in the United Arab Emirates and was approved by the Institutional Research and Ethics Committee. Subjects provided an informed consent by telephone for study participation.

Primary Outcome Variables

- Plasma TSH and free-T4 measured pre- and post-Ramadan.

\section{Independent Variables}

- Age and gender.

- Randomly assigned levothyroxine administration instruction group.

\section{Outcomes Modeling: Statistical Methodology}

Intent-to-treat effects were estimated in all analyses: randomized instruction groups were maintained during data analyses, regardless of reported compliance. Within-patient difference in plasma TSH and free-T4 levels across the pre- and post-Ramadan time points were modeled as a function of pre-Ramadan values and the randomly assigned levothyroxine administration instruction group.

We utilized mixed-effects models to estimate levothyroxine administration instruction group differences in endpoints, where a random patient-level intercept term was included to account for the repeated measures data structure. Although patients were randomized into groups to facilitate causal-effects estimates, additional statistical controls were utilized to further enhance the internal validity of our estimates. Specifically, groups were statistically equated on pre-Ramadan values on the endpoint under examination via the inclusion of a grand-median centered pre-Ramadan covariate. Additionally, group-specific time-by-pre-Ramadan value interaction covariates were included, thereby allowing pre-to post-Ramadan change to vary as a function of pre-Ramadan value uniquely for each group. The primary coefficients of interest describing group change pre-to-post were therefore estimated: (1) given a common pre-Ramadan value, and (2) after accounting for variability in change that occurs as a function of pre-Ramadan values. To illustrate model findings, this process was repeated using a grand 25 th and 75 th percentiles centered pre-Ramadan covariate. 
Table 1. Baseline characteristics of patients in the 3 randomization groups

\begin{tabular}{|c|c|c|c|c|c|c|}
\hline & \multicolumn{6}{|c|}{ Baseline patient characteristics } \\
\hline & group 1 & $N$ & group 2 & $N$ & group 3 & $N$ \\
\hline TSH, mIU/L, median (IQR) & $1.905(0.459-3.835)$ & 44 & $1.750(0.341-3.035)$ & 43 & $1.580(0.615-4.120)$ & 49 \\
\hline Free-T4, pmol/L, mean (SD) & $18.579(3.264)$ & 19 & $17.324(5.064)$ & 18 & $16.615(7.989)$ & 19 \\
\hline Males, \% & 18.33 & 60 & $16.00 \%$ & 50 & 33.87 & 62 \\
\hline Age, years, mean (SD) & $41.82(12.58)$ & 60 & $44.46(14.70)$ & 50 & $42.26(9.37)$ & 62 \\
\hline
\end{tabular}

Reference ranges: TSH 0.27-4.20 mIU/L; free-T4 10.0-23.0 pmol/L. SD, standard deviation; $N$, number of patients with data; IQR, interquartile range; $\mathrm{SD}$, standard deviation; free-T4, free thyroxine.

Table 2. TSH (mIU/L) pre- and post-Ramadan for the 3-randomization groups with at least 1 complete data point TSH change pre- to post-Ramadan, $\mathrm{mIU} / \mathrm{L}$

\begin{tabular}{|c|c|c|c|c|c|c|c|}
\hline \multirow[t]{2}{*}{ instruction groups } & \multirow[t]{2}{*}{$N$} & \multicolumn{2}{|c|}{ 25th percentile } & \multicolumn{2}{|c|}{ 50th percentile } & \multicolumn{2}{|c|}{ 75th percentile } \\
\hline & & pre & post & pre & post & pre & post \\
\hline (1) $30 \mathrm{~min}$ before Iftar & 50 & & 0.67 & & 1.29 & & $1.95^{*}$ \\
\hline (2) $3 \mathrm{~h}$ post-Iftar & 46 & 0.46 & $1.66^{* *}$ & 1.62 & 2.10 & 3.61 & 2.45 \\
\hline (3) 30 min before Suhur & 52 & & $1.30^{* *}$ & & 2.07 & & 2.78 \\
\hline
\end{tabular}

Equated pre-Ramadan values at 25th, 50th, and 75th percentiles and magnitude of change calculated using the mixed-effects regression model from online suppl. Table 1 . TSH reference range: $0.27-4.20 \mathrm{mIU} / \mathrm{L} .{ }^{*} p$ values examining pre-to-post-Ramadan change in TSH $<0.05 .{ }^{* *} p$ values examining pre-to-post-Ramadan change in TSH $<0.001$.

Mixed-effects regression models allow for any subject with at least 1 data point to contribute to the estimates by accounting for missing outcome data through Bayesian-weighted estimates that incorporate subject-specific reliabilities in the weighting scheme. Such methods have been shown to produce consistent and unbiased estimates of longitudinal effects in the presence of either missing completely at random or missing at random data [9].

In all instances, the criteria for determining statistical significance was a 2-tailed alpha $=0.05$. Prior to model estimation, TSH scores were log-transformed to reduce positive skew and better normalize the raw TSH data. Results were then transformed back to the original TSH scale to facilitate interpretation of findings. No transformation of free-T4 was performed due to the lack of skew in our sample data.

\section{Results}

The total number of patients who consented to enroll in this study was 148. Due to the long distance from the hospital and other patient-specific constraints, not all patients were able to attend the laboratory for blood collection before and after Ramadan. The numbers of patients

Levothyroxine Administration during Ramadan for the TSH endpoint with at least 1 data point were group $1(n=50)$, group $2(n=46)$, and group $3(n=52)$. The respective numbers for the free-T4 endpoint were 41,40 , and 41 . The median (interquartile range [IQR]) number of days for measurement of plasma TSH was 19.5 (4-43) days before Ramadan and 15.0 (6.25-22.8) days post-Ramadan, and for free-T4 were 15.0 (1-43) days pre- and 15.0 (7-22) days post-Ramadan.

Baseline characteristics of the 3 randomization groups are shown in Table 1 . The mean age $\pm S D$ of the whole cohort was $43.5 \pm 12.4$ (range 21.0-86.0) years with no significant differences between the 3 groups, and $78 \%$ of participants were female, but the group 3 had significantly more male than group $2(p$ value $=$ 0.035).

Mixed-effect model coefficients for TSH and free-T4 are shown in online suppl. Tables 1 and 2, respectively. Tables 2 and 3 show the estimated TSH and free-T4 values before and after Ramadan. Figure 1 presents a visual representation of Table 2 .

TSH increased in all 3 groups for patients at the 25th percentile and in groups 2 and 3 at the 50th percentile. 
Table 3. Free-T4 (pmol/L) pre- and post-Ramadan for the 3-randomization groups with at least 1 complete data point

\begin{tabular}{|c|c|c|c|c|c|c|c|}
\hline \multirow[t]{2}{*}{ instruction groups } & \multirow[t]{2}{*}{$N$} & \multicolumn{2}{|c|}{25 th percentile } & \multicolumn{2}{|c|}{ 50th percentile } & \multicolumn{2}{|c|}{ 75th percentile } \\
\hline & & pre & post & pre & post & pre & post \\
\hline (1) 30 min before Iftar & 41 & & 17.7 & & 18.19 & & 18.71 \\
\hline (2) $3 \mathrm{~h}$ post-Iftar & 40 & 15.80 & 17.11 & 18.30 & 18.01 & 20.93 & 18.94 \\
\hline (3) 30 min before Suhur & 41 & & 17.3 & & 17.42 & & $17.55^{*}$ \\
\hline
\end{tabular}

Equated pre-Ramadan values at 25th, 50th, and 75th percentiles and magnitude of change calculated using the mixed-effects regression model from online suppl. Table 2. Free-T4 reference range 10.0-23.0 pmol/L. FreeT4, free thyroxine. ${ }^{\star} p$ values for pre-to post-Ramadan free-T4 $<0.05$.

Fig. 1. Median plasma TSH (mIU/L) preand post-Ramadan modeled using mixedeffects regression for baseline TSH at 25th, 50 th, and 75 th percentiles. Reference range for plasma TSH $0.27-4.20 \mathrm{mIU} / \mathrm{L}$.

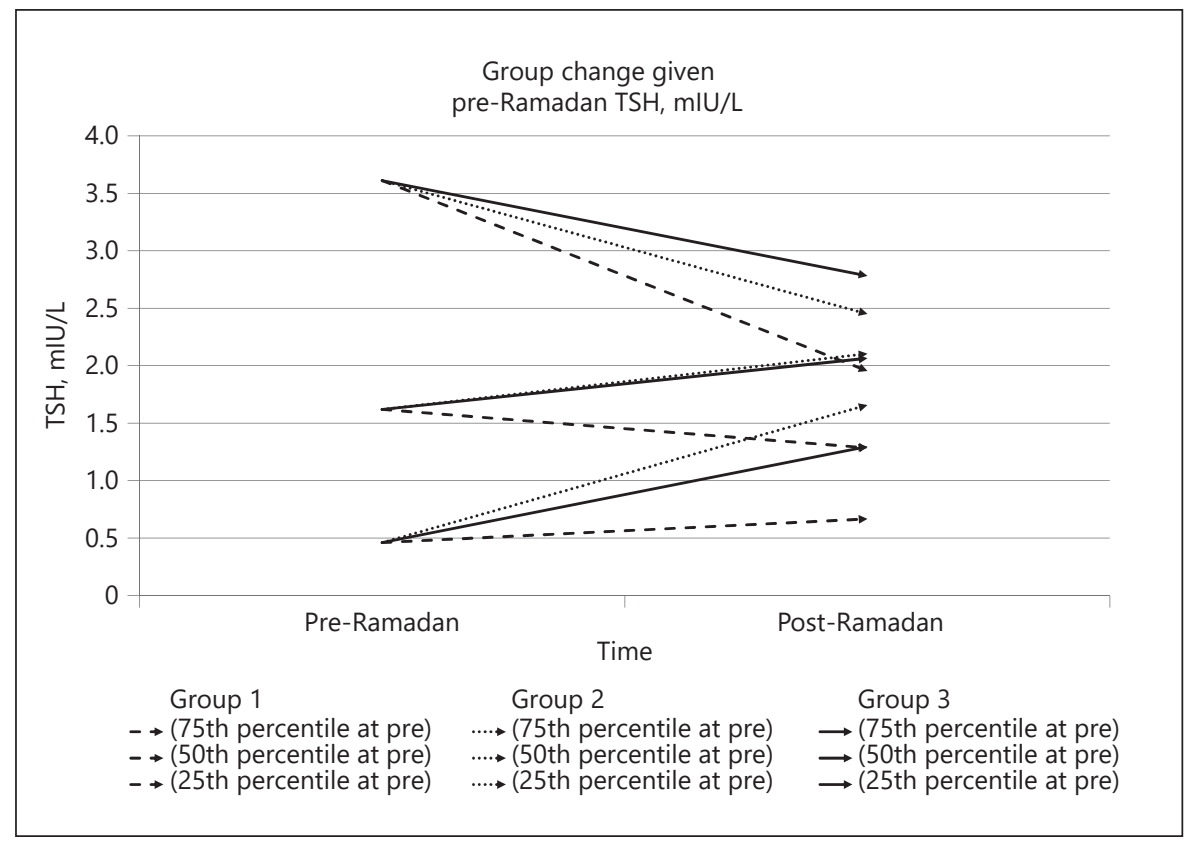

TSH decreased in group 1 for patients at the 50th percentile and in all 3 groups at the 75th percentile. However, within-group pre-to-post-Ramadan TSH changes were statistically significant only for groups 2 and 3 at the 25 th percentile ( $p$ value $<0.001$ ) and for group 1 at the 75 th percentile ( $p$ value 0.006 ) (Table 2; Fig. 1 ). To provide context for what these modeled changes imply at the group average level, descriptively speaking, the percentages of patients with a TSH above the normal reference range $(4.2 \mathrm{mIU} / \mathrm{L})$ pre-Ramadan were $22.72,13.95$, and $24.48 \%$ for groups 1, 2, and 3, respectively, while the respective percentages post-Ramadan were 11.76, 25.00, and $22.22 \%$.
Free-T4 increased in all 3 groups for patients at the 25 th percentile and decreased in all 3 groups for patients at the 50th and 75th percentiles. However, within-group pre-to-post-Ramadan free-T4 change was statistically significant only for group 3 at the 75 th percentile ( $p$ value $<0.05$ ) (Table 3).

Of the patients who responded to the post-Ramadan survey, 68/98 (69.4\%) complied with levothyroxine administration instructions and 53/92 (57.6\%) reported fasting every day during Ramadan (Table 4). There were no statistically significant differences in compliance rates or numbers of fasting days between the 3 groups. 
Table 4. Post-Ramadan survey results displaying compliance with levothyroxine administration instructions, patient-satisfaction with assigned levothyroxine administration time, and mean number of non-fasting days

\begin{tabular}{llllllll}
\hline & \multicolumn{2}{l}{ Compliance and satisfaction survey } & & \\
\cline { 2 - 8 } & group 1 & $N$ & group 2 & $N$ & group 3 & $N$ & total, $N$ \\
\hline Compliance, \% & 69.4 & 36 & 59.3 & 27 & 77.1 & 35 & 98 \\
Satisfaction, \% & 44.7 & 32 & 50.6 & 23 & 4.7 & 30 & 85 \\
Nonfasting days, mean (SEM) & $2.89(0.74)$ & 34 & $2.25(0.85)$ & 26 & $3.21(0.76)$ & 32 & 92 \\
\hline
\end{tabular}

$N$ denotes the number of respondents for each group. SEM, standard error of the mean.

\section{Discussion}

In this prospective randomized trial of levothyroxine-treated hypothyroid patients, our data suggest that changes in plasma TSH from pre-to-post-Ramadan are dependent on the baseline TSH and instructions concerning the time of levothyroxine administration. For patients at the 25th percentile pre-Ramadan, increases in plasma TSH were statistically significant in groups 2 and 3 but not in group 1, suggesting that group 1 had the best outcomes. Similar patterns were found at the 50th percentile, where plasma TSH descriptively increased in groups 2 and 3 but not in group 1. Finally, only group 1 showed a statistically significant decrease in TSH for patients at the 75th percentile pre-Ramadan, again suggesting that group 1 had the best outcomes. Additionally, these changes were associated with an $11 \%$ increase in the number of group 2 patients with plasma TSH above the upper limits of normal $(4.2 \mathrm{mIU} / \mathrm{L})$ compared to an $11 \%$ decrease in group 1 patients. Overall, though beneficial changes in TSH were statistically significant for group 1 only at the 75 th percentile, the consistent direction of group 1 results and the lack of significant negative changes in TSH by group 1 suggests that instructing patients to take levothyroxine $30 \mathrm{~min}$ before Iftar during Ramadan minimizes unfavorable changes in plasma TSH post-Ramadan, relative to instructing patients to take levothyroxine $3 \mathrm{~h}$ after Iftar or $30 \mathrm{~min}$ before Suhur.

The median number of days for free-T4 measurement post-Ramadan was 15.0 days (IQR 7-22) and may explain the lack of significant changes in free-T4 in most patients who had elevated post-Ramadan TSH levels as free-T4 changes precede those of TSH. In support of this, a small prospective study showed no significant decrease in freeT4 levels at 3 time points during Ramadan and 3 weeks post-Ramadan, despite significant increases in plasma
TSH at these intervals [1], whereas earlier measurement of free-T4 within 2 weeks of the end of Ramadan showed a significant decrease in plasma free-T4 in 1 prospective study [4], highlighting the importance of the timing of free-T4 measurement.

The reasons for the higher post-Ramadan plasma TSH when levothyroxine is taken $3 \mathrm{~h}$ post-Iftar and before Suhur, but not $30 \mathrm{~min}$ before Iftar are not clear, particularly because bedtime levothyroxine administration at $2,200 \mathrm{~h}$ in nonfasting subjects was shown in 1 prospective trial to be more efficacious and associated with significantly lower plasma TSH than taking levothyroxine 30 min before breakfast [10]. One potential reason for the higher plasma TSH in patients taking levothyroxine $3 \mathrm{~h}$ post-Iftar and before Suhur in our study could be an insufficient time period between food intake and levothyroxine administration, as previously suggested by 2 prospective studies [2, 3]. Karoli et al. [2] showed that taking levothyroxine at bedtime during Ramadan after an average of $66 \mathrm{~min}$ from the last meal led to an increase in plasma TSH. However, there was no significant change in TSH when the time form the last meal was extended to 85 min. Similarly, Sheikh et al. [3] showed that up to $75 \%$ of patients who took levothyroxine at least $2 \mathrm{~h}$ post-meal and $30 \mathrm{~min}$ pre-meal had a significant increase in plasma TSH post-Ramadan. In our study, patients in groups 2 and 3 were instructed to wait for a minimum of $3 \mathrm{~h}$ postmeal before taking levothyroxine, with 59.3 and $77.1 \%$ of patients reporting compliance with the instructions, but we are unable to verify this information.

It is equally uncertain why patients in group 1 had more favorable changes in plasma TSH post-Ramadan. In contrast to our findings, Dabbous et al. [4] demonstrated in a prospective randomized trial $(n=96)$ a significant increase in plasma TSH in patients taking levothyroxine 30 min before Iftar $(n=50)$ and before Suhur $(n=46)$. Differences in the baseline TSH and patient compliance, 
$30-35$ versus $69.4 \%$ in our study, may account for the discrepancies between the 2 studies.

Our study demonstrates a paradox between compliance and patient satisfaction with levothyroxine administration time (Table 4). While approximately $50 \%$ of 85 patients who were surveyed preferred taking levothyroxine $3 \mathrm{~h}$ post-Iftar, $45 \%$ before Iftar, and 5\% before Suhur, the respective compliance rates were $59.3 \%, 69.4 \%$, and $77.1 \%$. This paradox may be due to the ease and practicality of taking levothyroxine post-Iftar, as opposed to before Iftar or before Suhur.

We have previously shown in a retrospective analysis [5] that plasma TSH increases post-Ramadan irrespective of levothyroxine administration time, and that the increase was greater in older patients and in males. However, in our current prospective study, the increase in plasma TSH in groups 2 and 3 were not related to age or gender. Differences in study design and baseline TSH values may account for discrepancies between the 2 studies.

Strengths of this study include its prospective randomized design, with patient contact before and during Ramadan to reinforce compliance with levothyroxine administration. One potential limitation of this study is the timing of repeated plasma TSH and free-T4 measurement post-Ramadan, which vary widely: median 15.0 days (IQR 6.25-22.8) for TSH collection. Although plasma TSH remained significantly elevated beyond 3 weeks post-Ramadan in 1 prospective study [1], an earlier assessment of thyroid function tests post-Ramadan in our study may have shown a greater difference in TSH and free-T4 levels. In conclusion, our findings suggest that instructing patients to take levothyroxine $30 \mathrm{~min}$ before the Iftar meal may help mitigate unfavorable changes in plasma TSH that are otherwise expected post-Ramadan.

\section{Statement of Ethics}

The study was conducted ethically. The study protocol was approved by the Cleveland Clinic Abu Dhabi Research Ethics Committee on April 17, 2019 (Reference No. A2019-023), and subjects have given their informed consent before participating in the study. Clinical trial registration number: ISRCTN54141719 (https://doi.org/10.1186/ISRCTN54141719.).

\section{Conflict of Interest Statement}

The authors have no conflicts of interest to disclose.

\section{Funding Sources}

Test kits for plasma TSH and free-T4 were donated by Roche Diagnostics.

\section{Author Contributions}

In accordance with the ICMJE criteria for authorship, each of the listed co-authors made substantial contributions to this study as follows:

- S.E.-K. contributed to the study design, data collection, data analysis, and manuscript drafting.

- L.A. contributed to the study design, data collection, data analysis, and manuscript drafting.

- R.D. contributed to the study design, data collection, data analysis, and critical manuscript review.

- T.J.L. contributed to the study design, data analysis, and manuscript drafting.

- S.A.S. contributed to the study design, data collection, and critical manuscript review.

- F.M. contributed to the study design, data collection, and critical manuscript review.

- M.A. contributed to the study design, data collection, and critical manuscript review.

- A.K. contributed to data collection and critical manuscript review.

- Y.A. contributed to the study design and critical manuscript review.

\section{References}

1 Sajid KM, Akhtar M, Malik GQ. Ramadan fasting and thyroid hormone profile. J Pak Med Assoc. 1991 Sep;41(9):213-6.

2 Karoli R, Fatima J, Chandra A, Mishra PP. Levothyroxine replacement and Ramadan fasting. Indian J Endocrinol Metab. 2013 Mar; 17(2): 318-9.

3 Sheikh A, Mawani M, Mahar SA. Impact of ramadan fasting on thyroid status and quality of life in patients with primary hypothyroidism: a Prospective Cohort Study from Karachi, Pakistan. Endocr Pract. 2018 Oct 2;24(10):882-8.

4 Dabbous Z, Alowainati B, Darwish S, Ali H, Farook S, Al Malaheem $\mathrm{M}$, et al. A prospective study comparing two-time points of thyroid hormone replacement during the holy month of ramadan. Int J Endocrinol. 2019;2019:9843961.
5 El-Kaissi S, Dajani R, Lee-St John TJ, Ann Santarina S, Makia F, AlTakruri M, et al. Impact of lifestyle changes during ramadan on thyroid function tests in hypothyroid patients taking levothyroxine. Endocr Pract. 2020; 26(7):748-53.

6 Fish LH, Schwartz HL, Cavanaugh J, Steffes MW, Bantle JP, Oppenheimer JH. Replacement dose, metabolism, and bioavailability of levothyroxine in the treatment of hypothyroidism. Role of triiodothyronine in pituitary feedback in humans. N Engl J Med. 1987 Mar 26;316(13):764-70.

7 Wenzel KW, Kirschsieper HE. Aspects of the absorption of oral L-thyroxine in normal man. Metabolism. 1977 Jan;26(1):1-8.
8 Jonklaas J, Bianco AC, Bauer AJ, Burman KD, Cappola AR, Celi FS, et al. Guidelines for the treatment of hypothyroidism: prepared by the american thyroid association task force on thyroid hormone replacement. Thyroid. 2014 Dec;24(12):1670-751.

9 Rogosa D, Saner H. Longitudinal data analysis examples with random coefficient models. J Educ Behav Stat. 1995 Jun 1;20(2):14970.

10 Bolk N, Visser TJ, Kalsbeek A, van Domburg RT, Berghout A. Effects of evening vs. morning thyroxine ingestion on serum thyroid hormone profiles in hypothyroid patients. Clin Endocrinol. 2007 Jan;66(1):438. 